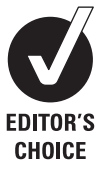

CHOICE

\title{
A comparison of two treatment protocols in the management of exercise-associated postural hypotension: a randomised clinical trial
}

\author{
Cameron Anley, ${ }^{1}$ Timothy Noakes, ${ }^{1}$ Malcolm Collins, ${ }^{1,2}$ Martin P Schwellnus ${ }^{1}$
}

${ }^{1} \mathrm{UCT} / \mathrm{MRC}$ Research Unit for Exercise Science and Sports Medicine, Department of Human Biology, Faculty of Health Sciences, University of Cape Town, Cape Town, South Africa

${ }^{2}$ South African Medical Research Council, Newlands, Cape Town, South Africa

\section{Correspondence to}

Dr Martin P Schwellnus, UCT/MRC Research Unit for Exercise Science and Sports Medicine, Department of Human Biology, Faculty of Health Sciences, University of Cape Town, 3rd Floor, Sports Science Institute of South Africa,Boundary Road, Newlands, Cape Town 7700, South Africa; martin. schwellnus@uct.ac.za

Accepted 1 April 2010 Published Online First 28 June 2010

\begin{abstract}
Objective To investigate which of two commonly used treatment protocols for exercise-associated postural hypotension (EAPH) resulted in earlier discharge from the medical facility.

Methods This randomised clinical field trial was undertaken at two Ironman Triathlon competitions and one ultra-distance footrace. All collapsed athletes admitted to the medical facilities were considered for the trial. Following clinical assessment and special investigations to confirm the diagnosis of EAPH, 28 athletes were randomly assigned to an oral fluid and Trendelenburg position (OT=14) or an intravenous fluid (IV=14) treatment group. Following admission fluid intake was recorded, and all athletes were assessed clinically (blood pressure, heart rate, level of consciousness) every 15 min until discharge criteria were met. The main measure of outcome was the time to discharge (min).
\end{abstract}

Results On admission, subjects in the OT and IV groups were similar with respect to age, systolic blood pressure, heart rate and serum sodium concentration. There were no significant differences in heart rate, systolic and diastolic blood pressure between groups and over time until discharge. The fluid intake during the treatment period was significantly greater in the IV group (IV $1045 \pm 185$ $\mathrm{ml}, 0 T 204 \pm 149 \mathrm{ml} ; \mathrm{p}<0.001)$. The average time to discharge for the $0 \mathrm{~T}$ group $(58 \pm 23 \mathrm{~min})$ was similar to that of the IV group ( $52.5 \pm 18 \mathrm{~min} ; \mathrm{p}=0.47)$.

Conclusion Endurance athletes with EAPH can be treated effectively using the Trendelenburg position and oral fluids and the administration of intravenous fluids does not reduce the time to discharge. The findings of this study support the hypothesis that EAPH is a result of venous pooling due to peripheral vasodilatation, rather than dehydration.

Due to the strenuous nature of ultra-endurance events, participants in these events are at risk of developing various medical conditions. ${ }^{1-7}$ One of the most common clinical presentations during and following an endurance event is that of the 'collapsed' athlete. Various medical conditions may lead to collapse in athletes, including cardiovascular, metabolic, neurological, musculoskeletal and respiratory causes. ${ }^{1-7}$

The most common cause for collapse is a condition referred to as exercise-associated postural hypotension (EAPH). ${ }^{38-10}$ The exact pathophysiology of EAPH is still widely debated in the literature, and currently two hypotheses have been proposed. The first hypothesis is that a combination of dehydration and hyperthermia causes circulatory failure, resulting in the collapse of the athlete. ${ }^{3} 511-14$
The second hypothesis is that pooling of blood occurs in the dilated venous system resulting in postural hypotension and thus collapse if generalised arterial vasoconstriction fails to occur immediately exercise terminates. ${ }^{29} 1015-17$

As a result of this ongoing debate, there is no clarity on how best to treat athletes with EAPH. Instead two protocols are used to manage athletes with EAPH in race medical facilities. These are (1) intravenous rehydration, ${ }^{51418}$ and (2) placing athletes in the Trendelenburg position with oral fluids given freely. ${ }^{2} 3719-21$

There appears to be a perception that athletes with EAPH recover quicker and are discharged earlier if treated with intravenous fluids, when compared with treating the athlete in the Trendelenburg position and administering oral fluids freely. However, to our knowledge, no controlled trials have investigated these two treatment protocols for EAPH in a systematic manner. In particular, no randomised clinical trials have compared these two treatment protocols in athletes with EAPH.

Therefore, the aim of this study was to compare which of the two commonly used treatment protocols for EAPH (Trendelenburg with freely available oral fluids or intravenous fluids) resulted in more rapid recovery and earlier discharge from the medical facility.

\section{METHODS}

\section{Type of study}

The study was conducted as a randomised clinical trial and the setting was that of a field trial.

\section{Subjects}

Subjects were recruited for this study from three ultra-endurance events, the 2006 and the 2007 South African Ironman Triathlon, and the 2006 Comrades ultramarathon. From these events, all the athletes who collapsed due to EAPH were considered potential subjects for this clinical field trial. The general methodology used to recruit subjects at these three events was similar and consisted of the following steps: pre-race athlete information, setting up research facilities in the medical tents that were integrated with the normal medical care facilities, informing all medical staff in the medical tents before the race of the nature of the study and the inclusion criteria for subjects, screening athletes in the triage area, and identifying athletes with EAPH using defined inclusion criteria as potential subjects, and obtaining informed consent from athletes with EAPH. 
Pre-race information about the study, including the purpose of the research and the methods, was available on the relevant race websites and was also provided during race registration. With agreement of the race medical directors, the protocols used were integrated with the race medical facilities. The medical staff where briefed on the protocols before the event.

Recruitment of the athletes took place on admission to the medical tent. This was done with the agreement of the race medical directors of all the events. As both treatment protocols are current medically acceptable protocols for the management of this condition, the race medical directors were satisfied that adequate medical care would still be provided by medical staff, irrespective of the protocol used. According to standard medical protocol, all athletes who presented with collapse at the medical facilities were placed in the supine position and an immediate baseline clinical assessment was completed by the attending medical staff. This assessment was designed to establish a diagnosis and identify any patient who may be severely ill and who may require urgent medical treatment. A diagnosis of EAPH was only confirmed once the following specific inclusion criteria were satisfied: (1) inability to stand or walk unaided after finishing the race (not during the race) as a result of light-headedness, faintness, dizziness or syncope; (2) normal level of consciousness and oriented; (3) systolic blood pressure greater than $100 \mathrm{~mm} \mathrm{Hg}$; (4) heart rate less than $100 \mathrm{bpm}$ and (5) body temperature less than $40^{\circ} \mathrm{C}$. The level of consciousness and orientation was assessed based on the following questions: (1) what is your name; (2) how old are you; (3) what is the date; (4) what race have you just completed and (5) where are you? If possible, a post-race body weight was obtained during this initial assessment.

The process of obtaining informed consent did not result in a delay of the treatment of these athletes. Consent was obtained on admission and confirmed again on discharge. Before the study, the protocol was approved by the Research Ethics Committee of the Faculty of Health Sciences at the University of Cape Town (ref numbers 425/2005, 185/2005 and 002/2007).

Once athletes were included in the study, members of the medical research team continued the management of these athletes. Thirty-two athletes (26 men and six women) were initially included in the trial.

\section{Experimental procedure}

Once athletes were entered into the trial, the following baseline medical assessment information was recorded: level of consciousness, heart rate and supine blood pressure. In addition, a venous blood sample was obtained to determine the blood glucose and serum sodium concentrations. Subjects were only included in the clinical trial if their serum sodium concentration was normal (135-148 mmol/l). The athletes were then randomly assigned to a treatment protocol based on the last digit of their race number.

Athletes with odd race numbers were recruited for the oral fluid and Trendelenburg position (OT) treatment protocol, which consisted of the following treatment details: (1) insertion of a venflon with the infusion of $50 \mathrm{ml}$ normal saline over $1 \mathrm{~h}$; (2) measurement of serum sodium concentration; (3) raising the athlete's legs and buttocks to $30^{\circ}$ and (4) advising the athlete that he or she may drink as much as desired. Athletes with even race numbers were recruited for the intravenous treatment protocol (IV), which consisted of the following treatment details: (1) insertion of a venflon with the infusion of $1000 \mathrm{ml}$ normal saline over $30 \mathrm{~min}$; (2) measurement of serum sodium concentrations; (3) the athletes remained in the supine position and (4) were advised that they may not drink during treatment. All fluid input was measured and documented in both groups.

Clinical re-assessments (level of consciousness, heart rate, supine blood pressure) were conducted every $15 \mathrm{~min}$ in both groups until discharge. Any athletes who had a change in these parameters had a repeat serum sodium concentration taken. Athletes were excluded from the study if their serum $\left[\mathrm{Na}^{+}\right]$ dropped below $135 \mathrm{mmol} / 1$. The treatment of the athletes who were removed from the trial was then taken over by the medical staff in the race medical tent. The reason for transfer from the trial was documented.

The primary endpoint for the clinical trial was discharge from the medical facility. At each 15 min interval, athletes were assessed for their eligibility for discharge from the medical facility. The criteria for discharge were: (1) normal mental state and (2) the athlete was able to stand and walk unassisted for $5 \mathrm{~m}$. The time of discharge (min) was recorded, and this was the main measure of outcome. If an athlete requested discharge, they were counselled and if they insisted, this was noted and they were discharged.

Four athletes were removed from the trial: one due to hypoglycaemia; one became confused and developed hyponatremia after 15 min while on the intravenous drip; one subject was randomly assigned to the oral trial but requested intravenous fluid after $30 \mathrm{~min}$ and one did not respond to $1000 \mathrm{ml}$ of intravenous fluids after $30 \mathrm{~min}$. The subject whp developed hyponatremia while receiving intravenous fluid was a 49 -yearold woman who presented with a serum $\left[\mathrm{Na}^{+}\right]$of $136 \mathrm{mmol} / 1$ on admission. After $15 \mathrm{~min}$ on the intravenous treatment (receiving approximately $500 \mathrm{ml}$ fluid) she became confused and her serum $\left[\mathrm{Na}^{+}\right]$had decreased to $134 \mathrm{mmol} / \mathrm{l}$. According to the protocol, she was then removed from the trial and transferred to the high-care medical treatment area. Of the final 28 athletes who completed the trial, 14 athletes were randomly assigned into the OT group (Trendelenburg and oral fluids) and 14 athletes were randomly assigned into the IV trial group (intravenous fluid).

\section{Measures of outcome}

The main outcome measure for this study was time to discharge from the medical tent (min). Additional measures of outcome were heart rate and blood pressure changes over time.

\section{Statistical analysis of the data}

Data were analysed with the STATISTICA version 7.0 statistical program. Any significant differences between the groups were determined by a one-way analysis of variance or $\chi^{2}$ analysis. When the overall F-value was significant, a Tukey's honest significance post-hoc test was used to determine specific differences. Statistical significance was accepted when $\mathrm{p}<0.05$.

\section{RESULTS \\ Subjects}

The age (mean $\pm S D)$ of the athletes in the OT group $(n=14)$ was $37.7 \pm 7.2$ years and that for the IV group ( $n=14$ ) was $36.5 \pm 7.1$ years. There was no significant difference between the ages of the two groups $(p=0.66)$. All the athletes were weighed on the morning of the race as they entered the transition zone. There were no significant differences in the pre-race weights 
(OT 73.6 $\pm 8.1 \mathrm{~kg}(\mathrm{n}=7)$ vs IV $76.0 \pm 6.2 \mathrm{~kg}(\mathrm{n}=5) ; \mathrm{p}=0.59)$ or gender distributions (OT 78.6\% men vs IV $92.9 \%$ men; $p=0.60$ ) between the two groups.

\section{Weather conditions}

The temperature, humidity and wind speeds for the three events were similar (table 1).

\section{Baseline assessment on admission to the medical facility}

There were no significant differences in the blood pressures, heart rates, axillary temperatures, serum $\left[\mathrm{Na}^{+}\right]$and plasma [glucose] of the athletes in the two protocol groups on admission to the medical facility following EAPH (table 2).

Pre-race and post-race body weights were obtained for 10 athletes (OT N=6; IV N=4). There were no significant differences in the absolute (data not shown) and relative pre-postrace body weight changes (table 2) of the athletes between the two groups on admission. Weight changes ranged from a weight loss of $4.9 \mathrm{~kg}$ to a weight gain of $3.4 \mathrm{~kg}$ in these athletes.

Table 1 Weather conditions for the ultra-endurance events at which the trial was performed

\begin{tabular}{llll}
\hline & Ironman 2006 & Ironman 2007 & Comrades Marathon 2006 \\
\hline Mean temperature $\left({ }^{\circ} \mathrm{C}\right)$ & 20 & 18 & 17 \\
Max temperature $\left({ }^{\circ} \mathrm{C}\right)$ & 21 & 21 & 22 \\
Min temperature $\left({ }^{\circ} \mathrm{C}\right)$ & 19 & 14 & 12 \\
Average humidity $(\%)$ & 75 & 56 & 75 \\
Maximum humidity (\%) & 88 & 94 & 94 \\
Minimum humidity $(\%)$ & 55 & 32 & 47 \\
Wind speed $(\mathrm{km} / \mathrm{h})$ & 28 & 17 & 4 \\
\hline
\end{tabular}

Table 2 Vital signs and blood results of athletes on admission

\begin{tabular}{lccl}
\hline & OT group (N=14) & IV group (N=14) & p Value \\
\hline SBP (mm Hg) & $110 \pm 11(91-130)$ & $106 \pm 8(89-115)$ & 0.21 \\
DBP (mm Hg) & $68 \pm 9(48-80)$ & $67 \pm 10(48-80)$ & 0.86 \\
Heart rate (bpm) & $81 \pm 10(60-99)$ & $90 \pm 14(42-102)$ & 0.79 \\
Serum [Na ${ }^{+}$] (mmol/l) & $139 \pm 3(135-147)$ & $141 \pm 5(136-150)$ & 0.18 \\
Plasma [glucose] (mmol/I) & $5.8 \pm 0.9(4.6-8.0)$ & $5.8 \pm 1.2(4.5-8.9)$ & 0.92 \\
Axillary temperature $\left({ }^{\circ} \mathrm{C}\right)^{*}$ & $36.1 \pm 0.9(34.8-37.5)$ & $35.9 \pm 0.5(35.1-37.5)$ & 0.62 \\
Pre-post-race body & $-3.5 \pm 3.0(-6.6-1.7)$ & $-2.2 \pm 4.9(-4.9-5.1)$ & 0.62 \\
weight changes $(\%)^{\dagger}$ & & & \\
\hline
\end{tabular}

Values are expressed as averages $\pm S D$ with the range in parenthesis.

*Temperature was only documented in 14 athletes (six in the oral fluid and

Trendelenburg position (OT) and eight in the intravenous fluid (IV) groups).

tRelative weight change was only documented in 10 athletes (six in the OT and four in the IV groups).

DBP, diastolic blood pressure; SBP, systolic blood pressure.
One athlete in each group had an increase in body weight after the race (OT $1.7 \%$ and IV 5.1\%). The rest of the athletes lost weight during the race. The greatest loss was $5.2 \%$ of pre-race weight, and this athlete, who was randomly assigned to the oral protocol, was discharged after $35 \mathrm{~min}$.

\section{Repeat assessments}

There were no significant differences in supine systolic and diastolic blood pressure and heart rate between the 28 athletes in the two groups or over the 15 min recovery periods (table 3 ). The number of athletes remaining after each 15 min recovery period is also indicated in table 3 .

The admission and final blood pressure and heart rate data of the athletes in the OT and IV protocol groups, respectively, are depicted in figure 1 . The discharge values are the last recorded vital signs before discharge and are therefore not necessarily recorded at the same time for each athlete. There were no significant differences in the changes in systolic blood pressure, diastolic blood pressure and heart rate from admission to discharge between the two groups.

\section{Fluid administration during recovery}

As shown in figure 2, the total volume of fluid $(\mathrm{ml})($ mean \pm SD) received by the OT group was $204 \pm 149 \mathrm{ml}(\mathrm{n}=11)$, and this was significantly less that that received by the IV group $1045 \pm 185$ $\mathrm{ml}(\mathrm{n}=12 ; \mathrm{p}<0.001)$. Data on fluid administration were incomplete in five patients.

\section{Time to discharge}

There was no significant difference in the time to discharge between the athletes in the IV group $(52.5 \pm 18 \mathrm{~min})$ compared with those in the OT group (58 $\pm 23 \mathrm{~min} ; \mathrm{p}=0.47)$ (figure 3 ).

\section{DISCUSSION}

The main finding of this study was that the time to discharge of athletes with EAPH is similar when treated with either intravenous fluids or when placed in the Trendelenburg position and given oral fluids freely.

It is clear that currently the most widely believed hypothesis for the pathophysiology of EAPH is that there is venous pooling of blood following exercise when the 'muscle pump' is removed on completion of exercise. ${ }^{2} 791517$ 19-23 The alternative hypothesis for the pathophysiology of EAPH is dehydration, which leads to hyperthermia and circulatory collapse as a result of a decreased central blood volume filling the heart. ${ }^{3} 511-13$

The main findings that support the hypothesis that venous pooling is the cause of EAPH is as follows: the rectal temperature

Table 3 Vital signs of the athletes during recovery

\begin{tabular}{|c|c|c|c|c|c|c|c|}
\hline & Admission & $15 \mathrm{Min}$ & 30 Min & 45 Min & 60 Min & 75 Min & 90 Min \\
\hline \multicolumn{8}{|c|}{ Supine SBP $(\mathrm{mm} \mathrm{Hg})$} \\
\hline ОТ & $110 \pm 11(14)(91-130)$ & $111 \pm 12(13)(93-130)$ & $109 \pm 9(11)(92-121)$ & $105 \pm 10(7)(93-120)$ & $110 \pm 7(5)(102-120)$ & $107 \pm 10(3)(95-115)$ & $123 \pm 0(1)(123-123)$ \\
\hline IV & $106 \pm 8(14)(89-115)$ & $104 \pm 11(14)(80-120)$ & $107 \pm 7(14)(96-121)$ & $105 \pm 7$ (9) (88-110) & $104 \pm 21(4)(78-129)$ & $97 \pm 4$ (3) (93-101) & $120 \pm 18(2)(107-132)$ \\
\hline ОТ & $68 \pm 9(14)(48-80)$ & $69 \pm 9(13)(52-85)$ & $66 \pm 6(11)(59-80)$ & $64 \pm 8(7)(50-73)$ & $68 \pm 10(5)(58-82)$ & $65 \pm 10$ (3) (53-71) & $78 \pm 0$ (1) (78-78) \\
\hline IV & $67 \pm 10(14)(48-80)$ & $64 \pm 9(14)(48-80)$ & $68 \pm 8(14)(52-80)$ & $68 \pm 8$ (9) (52-83) & $65 \pm 12$ (4) (50-78) & $63 \pm 6(3)(57-69)$ & $65 \pm 4(2)(62-67)$ \\
\hline IV & $90 \pm 14(14)(42-102)$ & $79 \pm 13(14)(63-110)$ & $74 \pm 9(14)(60-92)$ & $72 \pm 7$ (9) (65-88) & $68 \pm 3(4)(65-70)$ & $69 \pm 6(3)(65-75)$ & $74 \pm 8$ (2) (68-79) \\
\hline
\end{tabular}

Values are expressed as averages $\pm S D$ with the number of subjects $(N)$ and range in parenthesis.

DBP, diastolic blood pressure; IV, intravenous fluid group; OT, oral fluid and Trendelenburg position group; SBP, systolic blood pressure. 

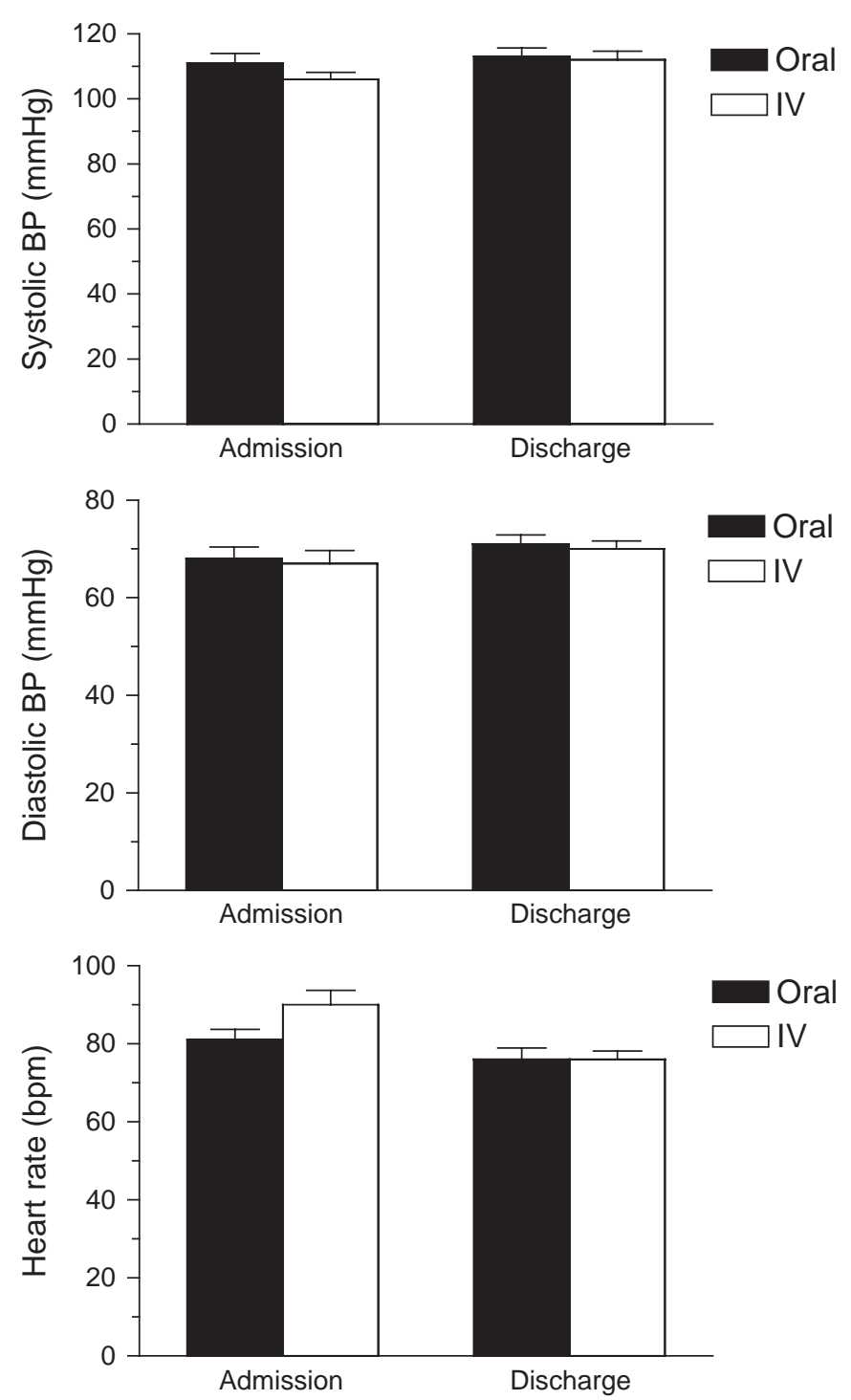

Figure 1 Comparison of supine systolic blood pressure (BP) (top panel), diastolic BP (middle panel), and heart rate on admission and discharge between the oral fluid and Trendelenburg position (oral) and intravenous fluid (IV) groups.

of the collapsed athlete is not significantly elevated in comparison with the non-collapsed runner; 291924 most collapsed athletes do not have clinical signs or biochemical confirmation of greater levels of dehydration than non-collapsed athletes; ${ }^{29}$ ${ }^{19}$ most (85\%) of the athletes who collapse do so upon completion of the race 2919 implying that physiological changes associated with the completion of exercise are causally related to the collapse; and symptoms of collapse can be rapidly reversed if the athlete is placed in the Trendelenburg position. ${ }^{29} 19$

The results of this study showed no difference in the time to recovery between the two protocols. This suggests that, in these athletes, raising the limbs in the Trendelenburg position is sufficient to restore plasma volume rather than by the rapid infusion of intravenous fluids. In this trial, athletes who received intravenous fluids were given on average five times as much fluid during their recovery period, yet did not recover more quickly than the oral group.

The results of this study therefore show that athletes with EAPH do not require intravenous fluid rehydration, but will respond as rapidly to treatment consisting of minimal oral

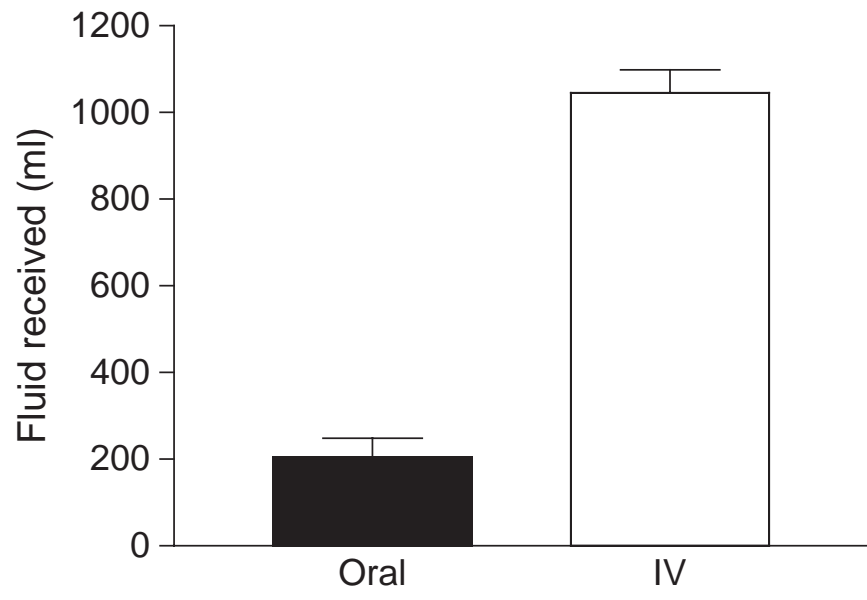

Figure 2 Volume of fluid the oral fluid and Trendelenburg position (oral) treatment group drank compared with the volume of intravenous fluid the intravenous fluid (IV) treatment group received while in the medical tent.

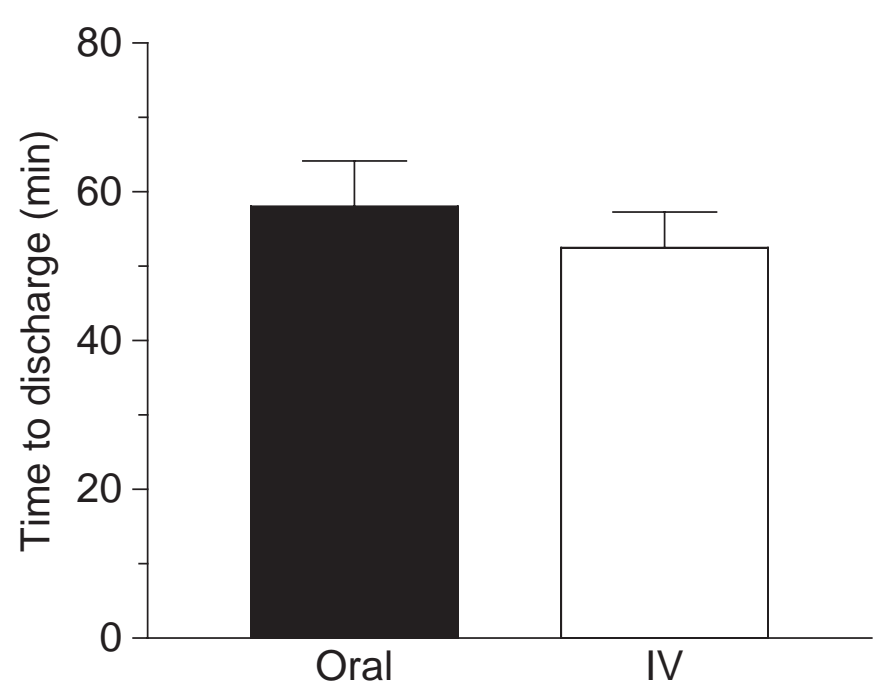

Figure 3 Time to discharge in the oral fluid and Trendelenburg position (oral) compares with the intravenous fluid (IV) treatment group.

fluid and raising the lower limbs (Trendelenburg position). Athletes with EAPH can adequately replace any fluid orally, provided they do not have any gastrointestinal disturbance, or cannot consume fluid for any other reason. The results of this study therefore support the hypothesis that EAPH is caused by pooling of blood in the lower limbs on completion of exercise.

The exact reason why some athletes are more predisposed to a persistent post-exercise state of low vascular resistance that is not matched by an increased cardiac output is currently the subject of ongoing research. First, immediately on cessation of exercise, there is a decrease in sympathetic nerve activity 2526 - the exact reason for this has not been fully explained. There may also be reduced adrenergic receptor responsiveness to sympathetic stimulation. Possible mechanisms for this may include downregulation of the receptors or by local vasodilator substances, such as nitric oxide. ${ }^{25}$ However, these mechanisms have not been investigated in humans. ${ }^{23} 2527$

A second possible mechanism for the venous pooling relates to arterial baroreflex function. The arterial baroreflex is a negative feedback reflex that regulates arterial pressure around a preset or operating point. The arterial baroreflex also 
establishes the prevailing systemic arterial pressure by setting the operating point. ${ }^{28}$ On this basis arginine vasopressin $(\mathrm{AVP})$, by its action on $\mathrm{V} 1$ receptors,${ }^{29}$ may contribute to the development of post-exercise hypotension. AVP enhances both cardiopulmonary and arterial baroreflex function, and lowers the operation point of the arterial baroreflex. ${ }^{28}$ AVP may enhance arterial baroreflex-induced inhibition of the sympathetic nervous system, which, as described earlier, decreases vasoconstrictor responsiveness and may therefore increase susceptibility to postural hypotension. ${ }^{29}$

Finally, the possible role of the histamine receptors, H1 and $\mathrm{H} 2$, in post-exercise hypotension has recently been suggested. ${ }^{30} 31$ In both studies the ingestion of antihistamines before exercise decreased the extent of post-exercise postural hypotension. ${ }^{3031}$ From the results of the two studies, it was concluded that histamine, by means of its stimulation of $\mathrm{H} 1$ and $\mathrm{H} 2$ receptors, may play a role in preventing the development of postural hypotension. The results of those studies also suggested that stimulation of $\mathrm{H} 1$ receptors may cause hypotension in the first $30 \mathrm{~min}$ post-exercise, whereas activation of $\mathrm{H} 2$ receptors has been shown to cause hypotension between 30 and $60 \mathrm{~min}$ post-exercise.

The diagnostic criteria for EAPH that were used in this study require some discussion. The two most important criteria when considering the athletes for the trial were: (1) a normal level of consciousness and (2) collapse that occurred after finishing the race. It has been well documented that athletes who are confused or collapse during the race are more likely to have any of a number of serious medical conditions. ${ }^{2} 41719212232$

The other major criteria for excluding subjects from this study were a temperature greater than $40^{\circ} \mathrm{C}$ (heat stroke), a blood glucose concentration less than $4 \mathrm{mmol} / 1$ (hypoglycaemia) and a serum sodium concentration less than 135 or greater than $148 \mathrm{mmol} / 1$ (dysnatraemia). The other exclusion criteria were a heart rate greater than $100 \mathrm{bpm}$ and a systolic blood pressure less than $100 \mathrm{~mm} \mathrm{Hg}$. Although these clinical signs may be present in an athlete with severe collapse, there is no evidence that these specific cut-off points distinguish more serious pathology from less serious pathology. For example, it is probable that blood pressure on standing was likely to have been very low and this could cause dizziness or syncope. Furthermore, blood pressure measured in the supine position may not reflect the extent of the blood pressure fall on standing.

However, due to the nature of the trial, data on pre-race and post-race body weight could not be collected from all the subjects. This is a limitation of the study. A further limitation of the study was that it was not possible to document the prerace blood pressure and heart rates. However, these vital signs, which were measured on admission to the medical tent, show that these athletes generally had a low blood pressure, and this is in keeping with findings reported from previous studies that have documented post-exercise hypotension. ${ }^{21732-34}$ The average heart rate of the athletes in both groups on admission was slightly elevated (81 bpm for OT and $90 \mathrm{bpm}$ for IV groups) but was not different between groups. The serum sodium concentrations and blood glucose concentrations of the athletes in both groups were within the normal range throughout the study, as abnormalities in these parameters were exclusion criteria.

The final limitation of this study was the small sample size. The reason for the small sample size was the difficult nature of the study. This study required careful planning and support from race organisers and medical staff. Furthermore, it was not possible to predict accurately the number of athletes who would collapse at events and would then be eligible for the trial. It therefore took three major events to obtain an adequate sample size for the study.

The time to discharge of the athletes is of interest to medical staff providing medical care at these events. In this study, 19 of 24 athletes were discharged within 60 min after admissionnine of 12 from the group who received oral fluids and 10 of 12 from the group given intravenous fluid. In this study, blood pressure remained stable over time and there was a small (not significant) decrease in heart rate over time during recovery. The comparison between admission and discharge blood pressure and heart rates showed that there was no significant difference during recovery in any of these vital signs between the two protocols. The discharge vital signs are the last recorded vital signs before discharge and thus are not recorded at the same time for each patient.

Finally, it is important to point out that there are no scientific criteria that justified the selection of criteria for discharge in this study - these were, however, based on the experiential evidence of sports physicians treating endurance athletes.

In summary, the result of this study show that time to discharge following EAPH is the same whether an athlete is given a large volume of intravenous fluid or is treated in the Trendelenburg position and given small volumes of oral fluids. These findings suggest that the cause of EAPH is not due to volume depletion (dehydration) but rather to venous pooling of blood in the lower limbs upon cessation of exercise. We also note that vital signs in the supine position did not change during the trials, whereas the ability to stand unaided improved progressively. This component of recovery is clearly not detectable when subjects are examined only in the lying position.

Funding This study was supported in part by funds from Discovery Health, the South African Medical Research Council (MRC) and the University of Cape Town Staff Research Fund. The final preparation of this study for publication was supported in part by the International Olympic Committee (IOC), research grant to the

\section{What is already known on this topic}

One of the most common clinical presentations during and following an endurance event is that of the 'collapsed' athlete. The most common cause for collapse is a condition referred to as EAPH. However, there is no clarity on how best to treat athletes with EAPH. The two protocols that are commonly used to manage athletes with EAPH in race medical facilities are intravenous rehydration and placing athletes in the Trendelenburg position with oral fluids given freely.

\section{What this study adds}

- The time to discharge from the medical facility following EAPH after an endurance event is the same whether an athlete is given a large volume of intravenous fluid or is treated in the Trendelenburg position and given small volumes of oral fluids.

- These findings suggest that the cause of EAPH is not due to volume depletion (dehydration) but rather to venous pooling of blood in the lower limbs upon cessation of exercise. 
Clinical Sports Medicine Group of the UCT/MRC Research Unit for Exercise Science and Sports Medicine of the University of Cape Town.

Competing interests None.

Ethics approval This study was conducted with the approval of the University of Cape Town Research Ethics Committee.

Patient consent Obtained.

Provenance and peer review Not commissioned; externally peer reviewed.

\section{REFERENCES}

1. Willix RD. Medical coverage for middle-distance triathlons. Ann Sport Med 1987:3:111-12.

2. Holtzhausen LM, Noakes TD. Collapsed ultraendurance athlete: proposed mechanisms and an approach to management. Clin J Sport Med 1997;7:292-301.

3. Roberts WO. A 12-year profile of medical injury and illness for the Twin Cities Marathon. Med Sci Sports Exerc 2000;32:1549-55.

4. Holtzhausen LM. Emergency medical cover for road races. Int Sportmed J 2003:4:1-13.

5. Adner MM, Scarlet JJ, Casey J, et al. The Boston Marathon medical care team: ten years of experience. Physician Sportsmed 1988;16:99-106.

6. Hew TD, Chorley JN, Cianca JC, et al. The incidence, risk factors, and clinical manifestations of hyponatremia in marathon runners. Clin J Sport Med 2003:13:41-7.

7. Brennan FH, O'Connor FG. Emergency triage of collapsed endurance athletes: a stepwise approach to on-site treatment. Phys Sportsmed 2005;33:28-35.

8. Speedy DB, Rogers IR, Noakes TD, et al. Diagnosis and prevention of hyponatremia at an ultradistance triathlon. Clin J Sport Med 2000;10:52-8.

9. Noakes TD. Reduced peripheral resistance and other factors in marathon collapse. Sports Med 2007;37:382-5.

10. Noakes TD. A modern classification of the exercise-related heat illnesses. J Sci Med Sport 2008;11:33-9

11. Armstrong LE, Hubbard RW, Kraemer WJ, et al. Signs and symptoms of heat exhaustion during strenuous exercise. Ann Sports Med 1987;3:182-8.

12. Hubbard RW Armstrong LE. Hyperthermia: new thoughts on an old problem. Phys Sportsmed 1989;17:97-113.

13. Eichner ER. Sacred cows and straw men. Phys Sportsmed 1991;13:24

14. Wyndham $\mathbf{C H}$, Strydom NB. The danger of an inadequate water intake during marathon running. S Afr Med J 1969;43:893-6.

15. Noakes TD. Exercise in the heat: old ideas, new dogmas. Int Sportmed J 2006;7:58-74.
16. Noakes TD. Dehydration during exercise: what are the real dangers? Clin J Sport Med 1995; 5:123-8

17. Sandell RC, Pascoe MD, Noakes TD. Factors associated with collapse during and after ultramarathon footraces: a preliminary study. Phys Sportsmed 1988;16:86-94.

18. Laird RH. Medical care at ultraendurance triathlons. Med Sci Sports Exerc 1989:21:S222-5

19. Speedy DB, Noakes TD, Holtzhausen LM. Exercise-associated collapse: postural hypotension, or something deadlier? Phys Sportsmed 2003;31:23-9.

20. Pyne S. Intravenous fluids post marathon: when and why? Sports Med 2007; 37:434-6.

21. Mayers LB, Noakes TD. A guide to treating ironman triathletes at the finish line. Phys Sportsmed 2000;28:35-50

22. Blue JG, Pecci MA. The collapsed athlete. Orthop Clin North Am 2002:33:471-8.

23. Krediet CT, Wilde AA, Wieling W, et al. Exercise related syncope, when it's not the heart. Clin Auton Res 2004;14(Suppl 1):25-36.

24. Laird RH. Medical complications during the Ironman Triathlon World Championship 1981-1984. Ann Sports Med 1987;3:113-16.

25. Halliwill JR, Taylor JA, Eckberg DL. Impaired sympathetic vascular regulation in humans after acute dynamic exercise. J Physiol (Lond) 1996;495:279-88.

26. Kulics JM, Collins HL, DiCarlo SE. Postexercise hypotension is mediated by reductions in sympathetic nerve activity. Am J Physiol 1999;276:H27-32.

27. Halliwill JR. Mechanisms and clinical implications of post-exercise hypotension in humans. Exerc Sport Sci Rev 2001;29:65-70.

28. Chandler MP, Rodenbaugh DW, DiCarlo SE. Arterial baroreflex resetting mediates postexercise reductions in arterial pressure and heart rate. Am J Physiol 1998;275:H1627-34.

29. Collins HL, Rodenbaugh DW, DiCarlo SE. Central blockade of vasopressin V(1) receptors attenuates postexercise hypotension. Am J Physiol Regul Integr Comp Physiol 2001;281:R375-80.

30. McCord JL, Beasley JM, Halliwill JR. H2-receptor-mediated vasodilation contributes to postexercise hypotension. J App/ Physiol 2006;100:67-75.

31. Lockwood JM, Wilkins BW, Halliwill JR. H1 receptor-mediated vasodilatation contributes to postexercise hypotension. J Physiol (Lond) 2005;563:633-42.

32. Holtzhausen LM, Noakes TD. The prevalence and significance of postexercise (postural) hypotension in ultramarathon runners. Med Sci Sports Exerc 1995;27:1595-601.

33. Eichna LW, Horvath SM, Bean WB. Post-exertional orthostatic hypotension. Am J Med Sci 1947;213:641-54.

34. Gratze G, Rudnicki R, Urban W, et al. Hemodynamic and autonomic changes induced by Ironman: prediction of competition time by blood pressure variability. J App/ Physio/ 2005;99:1728-35 